\title{
Early changes in bacterial communities in wound tissues of Pinus massoniana after inoculation with Bursaphelenchus xylophilus
}

\author{
Jinyan LIU ${ }^{1,2}$, Songqing Wu ${ }^{1}$, Xia Hu $^{1}$, Wanfeng XIE ${ }^{2}$, Xiuping HuANG ${ }^{1,2}$, \\ Guanghong LIANG ${ }^{1}$, Dan GuO ${ }^{1}$, Jieqin WU ${ }^{1}$ and Feiping ZHANG ${ }^{1, *}$ \\ ${ }^{1}$ College of Forestry, Fujian Agriculture and Forestry University, Fuzhou 350002, P.R. China \\ ${ }^{2}$ Jinshan College of Fujian Agriculture and Forestry University, Fuzhou 350002, P.R. China
}

Received: 22 March 2020; revised: 18 May 2020 Accepted for publication: 18 May 2020; available online: 8 July 2020

\begin{abstract}
Summary - The bacterial communities in the wound tissues of Pinus massoniana were analysed by 16S rDNA amplicon sequencing. The results showed that the bacterial richness and diversity changed remarkably whether the wound was inoculated with pine wood nematode (PWN; Bursaphelenchus xylophilus) or not after $12 \mathrm{~h}$. However, the predominant bacteria Stenotrophomonas, Burkholderiaceae, Pseudomonas, Serratia and Delftia, introduced by PWN in the wound tissues, changed within 6 h. After 6 h of PWN inoculation, the most abundant genus associating with PWN, Stenotrophomonas, failed to colonise the wound tissues, and the abundance of Delftia decreased, while the other representative bacteria, Burkholderiaceae, Pseudomonas and Serratia, from the PWN were markedly enriched. In addition, our study is the first to report the association of Serratia liquefaciens with PWN. Predicted functional analyses using the Tax4Fun tool showed that the alterations in bacterial composition also led to shifts in their functional pathways, especially after $12 \mathrm{~h}$ of PWN inoculation. These findings clarified that the bacteria carried by PWN were responsible for the alterations in bacterial communities in the wound tissues and will shed light on the invasion mechanism of PWN.
\end{abstract}

Keywords - 16S rDNA, bacterial diversity, Burkholderiaceae, Delftia, pine wilt disease, pine wood nematode, Pseudomonas, Serratia, Serratia liquefaciens, Stenotrophomonas.

Pine wilt disease (PWD) is one of the most destructive diseases of pine trees in the world. The causal agent of the disease was initially confirmed as Bursaphelenchus xylophilus (Steiner \& Buhrer, 1934), the pine wood nematode (PWN), by Kiyohara \& Tokushige (1971) and for years it was thought to be the only pathogenic agent (Mamiya, 1975, 1983; Nickle et al., 1981; Fukuda et al., 1992). However, some scientists studying the mechanisms of PWD attributed the rapid wilting of pine trees to wilt toxins, and the toxins were probably produced by nematode-associated bacteria (Oku et al., 1979, 1980; Kawazu et al., 1996; Han et al., 2003; Zhao et al., 2003; Tan \& Feng, 2004; Guo et al., 2006; Yin et al., 2007). As a result, many experiments have been conducted on these bacteria, including surface bacteria and endobacteria of PWN (Zhao et al., 2000; Wu et al., 2013; Tian et al., 2015), as well as bacteria associated with the beetle vectors (Vicente et al., 2013a; Alves et al., 2016) and hosts of PWN (Proença et al., 2017a), aiming to clarify

* Corresponding author, e-mail: fpzhang1@163.com the origin and exact role of bacteria in PWD. As reviewed by Proença et al. (2017b), the bacteria Pseudomonas, Burkholderia, Serratia, Ewingella and Enterobacter were most commonly reported to be associated with PWD in China, Korea, Portugal and the USA, and other genera, such as Stenotrophomonas, Bacillus and Pantoea, were also carried by the PWN. However, despite the intense research and abundant information about the possible functions of these bacteria, the role of associated bacteria in PWD has mostly been tested in vitro, and when these bacteria come into play in PWD progression remains unclear.

In the wild, the PWN is transmitted from tree to tree by insect vectors, mostly from the genus Monochamus (Coleoptera: Cerambycidae), including Monochamus alternatus in East Asia (Mamiya \& Enda, 1972; Lee et al., 1990; Ning et al., 2004), M. carolinensis in North America (Linit et al., 1983) and M. galloprovincialis in Portugal (Naves et al., 2001), through feeding wounds (Linit, 
1990) or oviposit wounds (Edwards \& Linit, 1992). Afterwards, the PWN moves from the trachea to the tail tip of the vectors and is finally transmitted to the wounds of pine twigs (Aikawai \& Tiogashi, 1998), where a new infection cycle begins. Nevertheless, the nematodes hardly migrate or migrate slowly on the wound surface (Tamura, 1984) because most of them are trapped in the sticky resin exuded by epithelial cells on the wound surface (Zhao, 2008) and have to survive harmful secondary metabolites produced by defence mechanisms of the host (Cheng et al., 2013) before successfully invading pine tissues. In this initial stage, some bacteria in the wound may already begin to react against the defence metabolites of the host and help PWN tolerate and overcome the resistance of the host. Therefore, determining what species of bacteria are the pioneers that play a role in this stage is important for illuminating the invasion mechanism of PWN.

To answer the questions mentioned above, the aim of this study was to analyse the changes in bacterial communities in wound tissues caused by inoculation of PWN in the initial stage using 16S rDNA amplicon sequencing, aiming to clarify the bacterial pioneers that would affect the successful invasion of PWN.

\section{Materials and methods}

\section{EXPERIMENTAL MATERIALS}

A virulent PWN strain was initially isolated from naturally infected Pinus massoniana (Fujian, P.R. China). After morphological identification, the nematodes were propagated on Pestalotiopsis sp. (Xie et al., 2017a, b; Sriwati et al., 2008) cultured on PDA medium at $28^{\circ} \mathrm{C}$ for 7 days. The harvested nematodes were then collected using a Baermann funnel. Large quantities of nematodes were obtained based on repetitive propagation using this method and stored at $4^{\circ} \mathrm{C}$ until use. Three-year-old $P$. massoniana seedlings with similar growing conditions (height, 70-80 cm; diam., $1 \mathrm{~cm}$ ), planted in the Institute of Forest Protection in Fujian Agriculture and Forestry University, China, were used in the inoculation experiment.

\section{INOCULATION AND SAMPLING}

The cultured nematodes were rinsed three times with sterile deionised water before use and adjusted to $30 \mu \mathrm{l}$ nematode suspension (approximately 1000 nematodes) in each $0.5 \mathrm{ml}$ PCR tube; this solution was then directly pipetted onto the artificial wounds of the seedlings. Each seedling had five artificial wounds that were generated by scraping the bark with a razor blade. The longitudinal and axial lengths of the wounds were $3 \mathrm{~cm}$ and $0.5 \mathrm{~cm}$, respectively, and the wounds were $5 \mathrm{~cm}$ apart from each other. The plants inoculated with the same amount of sterile water were used as controls. To distinguish the origin of bacteria in the wound tissues after inoculation, the bacterial communities carried by the PWN and the communities that originally existed in the cortex tissues of healthy P. massoniana seedlings were detected.

As most of the nematodes were trapped on the wound surface for approximately 6-12 $\mathrm{h}$ according to different host and inoculum densities (Jin, 2007; Zhang et al., 2007; Li \& Ye, 2008; Su et al., 2008), we chose 6 h and $12 \mathrm{~h}$ as the experimental time points. The tissues of the wounds, mostly the cortex, were collected 6 and $12 \mathrm{~h}$ after inoculation and then stored at $-80^{\circ} \mathrm{C}$ until use, as were the corresponding two control groups. The cortex tissues of healthy $P$. massoniana seedlings were collected immediately after the bark had been scraped and the rest of the cultured nematodes (three tubes with $0.5 \mathrm{ml}$ nematode precipitate in each) were rinsed three times with sterile deionised water before storing at $-80^{\circ} \mathrm{C}$. Because of the limited amount of cortex tissues from one single wound, a mixture of 15 cortex tissues from 15 wounds (three seedlings) was treated as a treatment sample, and each treatment had three replicates (nine seedlings). Thus, a total of 45 pine seedlings were used in this research, with nine seedlings in each group (two experimental groups, two control groups and one group of healthy pine tissues). The tools used above were all decontaminated before use, and the experiment was conducted at $28^{\circ} \mathrm{C}$ in a glasshouse.

\section{DNA EXTRACTION}

Total genomic DNA of the samples was extracted using the CTAB method (Lutz et al., 2011), and the concentration and purity were detected by $1 \%$ agarose gel electrophoresis. Afterwards, the DNA products were diluted to $1 \mathrm{ng} \mu \mathrm{l}^{-1}$ with sterile water before PCR amplification.

\section{PCR AMPLIFICATION}

To study the diversity and composition of bacteria in the wound tissues, the distinct V3-V4 regions of $16 \mathrm{~S}$ rDNA were PCR amplified with specific barcoded primers: 341F (5'-CCTAYGGGRBGCASCAG-3') and 806R (5'GGACTACNNGGGTATCTAAT- $3^{\prime}$ ). PCR was performed 
in a $30 \mu \mathrm{l}$ volume, containing $15 \mu \mathrm{l}$ Phusion ${ }^{\circledR}$ HighFidelity PCR Master Mix (New England Biolabs), $0.1 \mu \mathrm{M}$ each primer and $10 \mathrm{ng}$ template DNA. Thermal cycling consisted of initial denaturation at $98^{\circ} \mathrm{C}$ for $1 \mathrm{~min}$, followed by 30 cycles of denaturation at $98^{\circ} \mathrm{C}$ for $10 \mathrm{~s}$, annealing at $55^{\circ} \mathrm{C}$ for $30 \mathrm{~s}, 72^{\circ} \mathrm{C}$ for $30 \mathrm{~s}$ and a final extension at $72^{\circ} \mathrm{C}$ for $5 \mathrm{~min}$.

\section{GENE LIBRARY PREPARATION AND SEQUENCING}

PCR products were detected using $2 \%$ agarose gel electrophoresis, and then purified with a GeneJET Gel Extraction Kit (Thermo Scientific). Sequencing libraries were generated using the Ion Plus Fragment Library Kit 48 rxns (Thermo Scientific) following the manufacturer's recommendations. After assessing the library quality on the Qubit@ 2.0 Fluorometer (Thermo Scientific), the PCR products were sequenced on an Ion S5 ${ }^{\mathrm{TM}}$ XL platform, and $600 \mathrm{bp}$ single-end reads were generated.

\section{BIOCHEMICAL ANALYSIS}

Raw reads were obtained after single-end reads were assigned to samples based on their unique barcode and truncated by removing the barcode and primer sequences. Quality filtering of the raw reads was performed under specific filtering conditions to obtain high-quality clean reads according to the Cutadapt (Version 1.9.1, http:// cutadapt.readthedocs.io/en/stable/) (Martin, 2011) quality control process. Subsequently, clean reads were finally obtained after comparison with the Silva database (https://www.arb-silva.de/) (Quast et al., 2012) using the UCHIME algorithm (http://www.drive5.com/usearch/ manual/uchime_algo.html) (Edgar et al., 2011) to detect and remove the chimeric sequences.

Sequence analysis was performed by UPARSE software (Version 7.0.1001, http://drive5.com/uparse/) (Edgar, 2013). Sequences with $\geqslant 97 \%$ similarity were clustered to the same operational taxonomic units (OTUs). A representative sequence for each OTU was screened for further annotation, and taxonomic information was annotated by means of the Silva database based on the MOTHUR algorithm.

To assess microbial diversity with or without infection by PWN, alpha diversity was calculated, including observed species, Chao 1, Shannon, Simpson and good coverage, all of which were calculated with QIIME (Version 1.7.0) (Caporaso et al., 2010) and displayed with R software (Version 2.15.3).
To evaluate the differences in species complexity among samples, beta diversity and unweighted UniFrac diversity were analysed by QIIME software (Version 1.7.0) (Caporaso et al., 2010). The significant differences in bacterial community structure among samples were analysed using MetaStat (White et al., 2009) and LEfSe (Segata et al., 2011). The functional diversity of bacteria in each group was predicted by Tax4Fun (Aßhauer et al., 2015).

\section{Results}

\section{SEQUENCE ANALYSIS}

The bacterial reads of the samples inoculated with B. xylophilus after 6 and $12 \mathrm{~h}$ (indicated as Bx6h and $\mathrm{Bx} 12 \mathrm{~h}$, respectively), and their control groups inoculated with sterile water (C6h and $\mathrm{C} 12 \mathrm{~h}$, respectively), as well as healthy wound tissues ( $\mathrm{Pm})$ and B. xylophilus $(\mathrm{Bx})$, were cut and filtered. After quality control, a total of 1129409 bp clean reads, which clustered in 1157 OTUs, were obtained. Among these OTUs, only two (0.17\%) OTUs could not be annotated, whereas 1081 (93.43\%), 1044 (90.23\%), (83.32\%), 964 (74.33\%), 567 (49.01\%) and $113(9.77 \%)$ OTUs were annotated at the phylum, class, order, family, genus and species levels, respectively. The rarefaction curve (Fig. 1) for each group nearly reached saturation, indicating that the sequence data were sufficient and representative.

\section{BACTERIAL DIVERSITY ANALYSIS}

To assess the possible alterations in bacterial diversity caused by PWN inoculation, alpha diversity was analysed based on observed species, Chao 1 (richness), Shannon index and Simpson index (diversity) (Table 1). All indices indicated that the bacterial richness and diversity in Group Bx6h were comparable with those of its control group (C6h), while Group Bx12h had significantly lower richness and diversity indices compared to the control group (C12h) $(P<0.05$, Wilcox). Even in the two control groups, the bacterial richness and diversity changed, with the longer time group having greatly increased indices. Based on the sequencing data, we found that the main genera that increased in abundance (Group $\mathrm{C} 12 \mathrm{~h}$ compared to Group C6h) was Rhizobacter and other unidentified genera. Moreover, compared to all the other groups, Group Bx had the lowest bacterial richness and diversity $(P<0.05$, Wilcox $)$. 


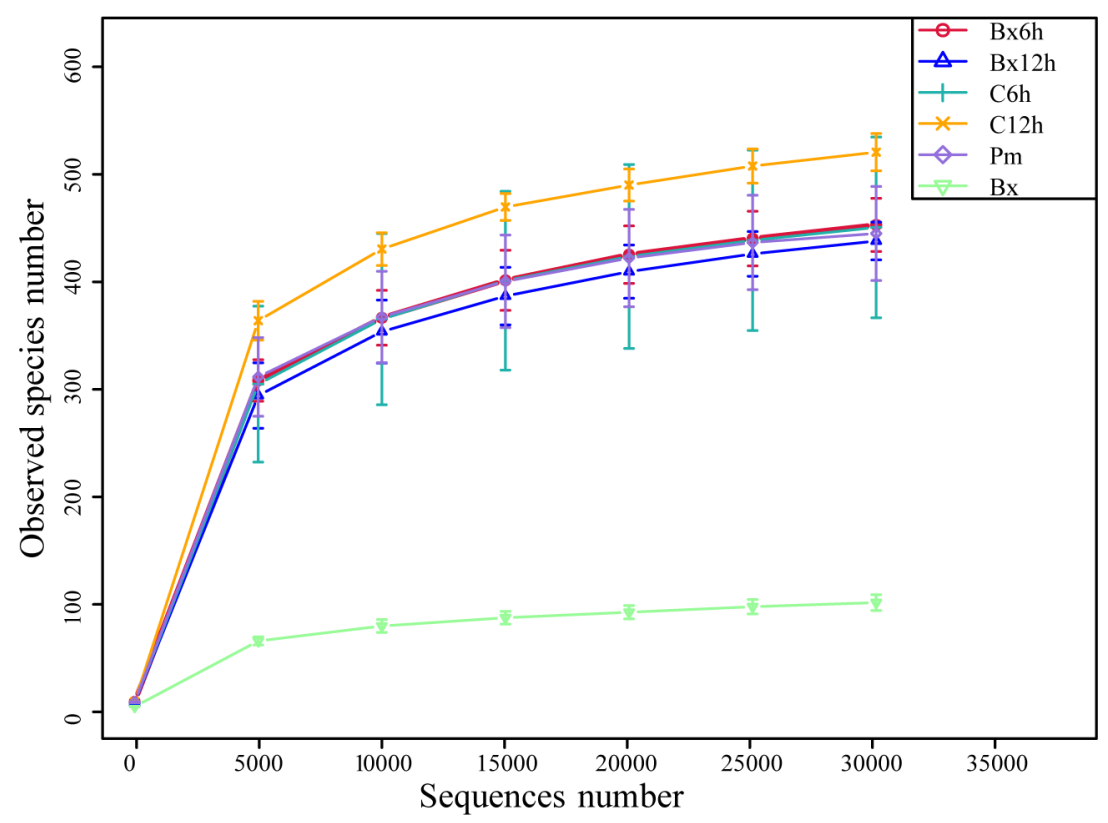

Fig. 1. Rarefaction curves of observed species from the $6 \mathrm{~h}$ pine wood nematode (PWN; Bursaphelenchus xylophilus)-inoculation group (Bx6h), the $12 \mathrm{~h}$ PWN-inoculation group (Bx12h), their corresponding control groups (C6h, C12h), healthy cortex of Pinus massoniana $(\mathrm{Pm})$ and B. xylophilus $(\mathrm{Bx})$ group.

Table 1. Alpha diversity indices of bacteria associated with the $6 \mathrm{~h}$ pinewood nematode (PWN; Bursaphelenchus xylophilus)-inoculation group (Bx6h), the $12 \mathrm{~h}$ PWN-inoculation group (Bx12h), their corresponding control groups (C6h, C12h), healthy cortex of Pinus massoniana $(\mathrm{Pm})$ and B. xylophilus $(\mathrm{Bx})$ group.

\begin{tabular}{llllll}
\hline Group & $\begin{array}{c}\text { Observed } \\
\text { species }\end{array}$ & Shannon & Simpson & Chao1 & Coverage \\
\hline Bx6h & 453 & 6.596 & 0.979 & 497.865 & 0.998 \\
C6h & 450 & 6.505 & 0.974 & 486.561 & 0.998 \\
Bx12h & $438 *$ & $6.100 *$ & $0.960 *$ & $488.648 *$ & 0.998 \\
C12h & 520 & 7.103 & 0.986 & 576.471 & 0.998 \\
Pm & 445 & 6.338 & 0.969 & 465.311 & 0.998 \\
Bx & $101 * *$ & $2.961 * *$ & $0.781 * *$ & $121.701 * *$ & 0.999 \\
\hline
\end{tabular}

* Statistically different $(P<0.05$, Wilcox $)$ compared to its control group.

** Statistically different $(P<0.05$, Wilcox $)$ compared to all the other groups.

\section{BACTERIAL COMMUNITY COMPOSITION}

To gain insights into the differences in bacterial communities, we further analysed the individual bacterial taxa in each group and presented the results according to the clusters of Unweighted Pair-group Method with Arithmetic Means (UPGMA) Clustering. As shown in Figure 2, the PWN-inoculated groups (Bx6h, Bx12h) shared a similar relative abundance of bacterial phyla and clustered together, while the bacterial phyla of Group Bx that we used for inoculation were distinctive from the other five groups.

In Group Bx, a total of 160 OTUs were detected, with Proteobacteria (mean \pm standard error; $98.3 \pm 0.45 \%$ ) being the most abundant phylum, followed by Bacteroidetes $(1.3 \pm 0.31 \%)$. At the class level, $\gamma$-proteobacteria dominated most of the total reads, with a percentage of $96.0 \pm 0.56 \%$, and $\alpha$-proteobacteria $(2.4 \pm 0.30 \%)$ ranked second (Fig. 3A). At the order level, Xanthomonadales $(36.3 \pm 3.96 \%)$ ranked first, followed by unidentified $\gamma$-proteobacteria $(35.6 \pm 6.00 \%)$ and Pseudomonadales $(19.2 \pm 1.35 \%)$ (Fig. 3B). There were five representative families that occupied $>1 \%$ of the total reads: Xanthomonadaceae $(36.2 \pm 3.97 \%)$, Burkholderiaceae $(35.6 \pm 6.02 \%)$, Pseudomonadaceae $(19.2 \pm 1.36 \%)$, Enterobacteriaceae $(4.8 \pm 0.42 \%)$ and Sphingomonadaceae $(1.6 \pm 0.19 \%)$ (Fig. 3C). The most abundant genus was Stenotrophomonas (36.2 $\pm 3.97 \%)$, followed by unidentified Burkholderiaceae $(31.9 \pm 5.98 \%)$, Pseudomonas $(19.2 \pm 1.36 \%)$, Serratia $(3.7 \pm 0.23 \%)$, Delftia $(1.6 \pm$ $0.40 \%)$, Novosphingobium $(1.4 \pm 0.22 \%)$ and Chryseobacterium $(1.0 \pm 0.30 \%)$ (Fig. 3D). The relative abundance of other genera was less than $1 \%$. 


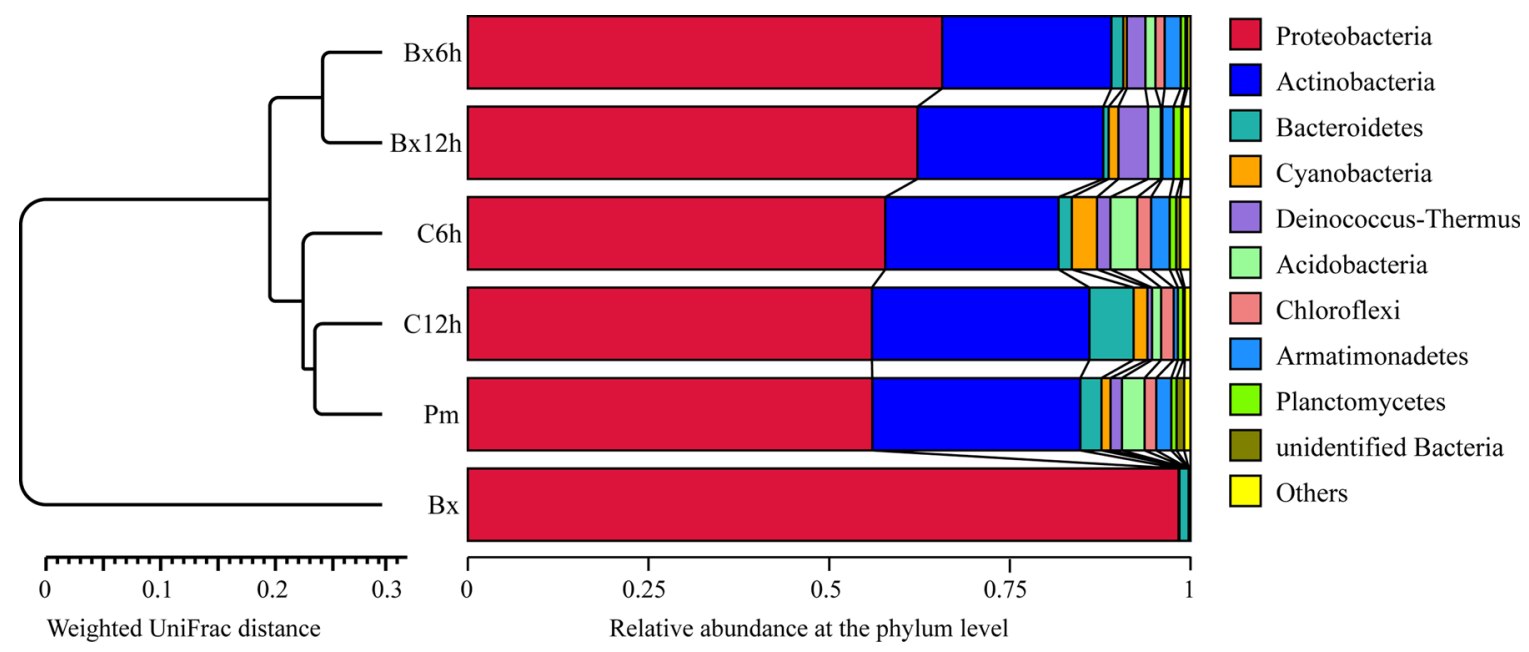

Fig. 2. Relative abundance at the phylum level using the unweighted pair-group method with arithmetic means (UPGMA) clustering. $\mathrm{Bx} 6 \mathrm{~h}$, the $6 \mathrm{~h}$ pine wood nematode (PWN; Bursaphelenchus xylophilus)-inoculation group; Bx12h, the $12 \mathrm{~h}$ PWN-inoculation group; $\mathrm{C} 6 \mathrm{~h}$, control group of $\mathrm{Bx} 6 \mathrm{~h} ; \mathrm{C} 12 \mathrm{~h}$, control group of $\mathrm{Bx} 12 \mathrm{~h}$; Pm, healthy cortex of Pinus massoniana; Bx, B. xylophilus.
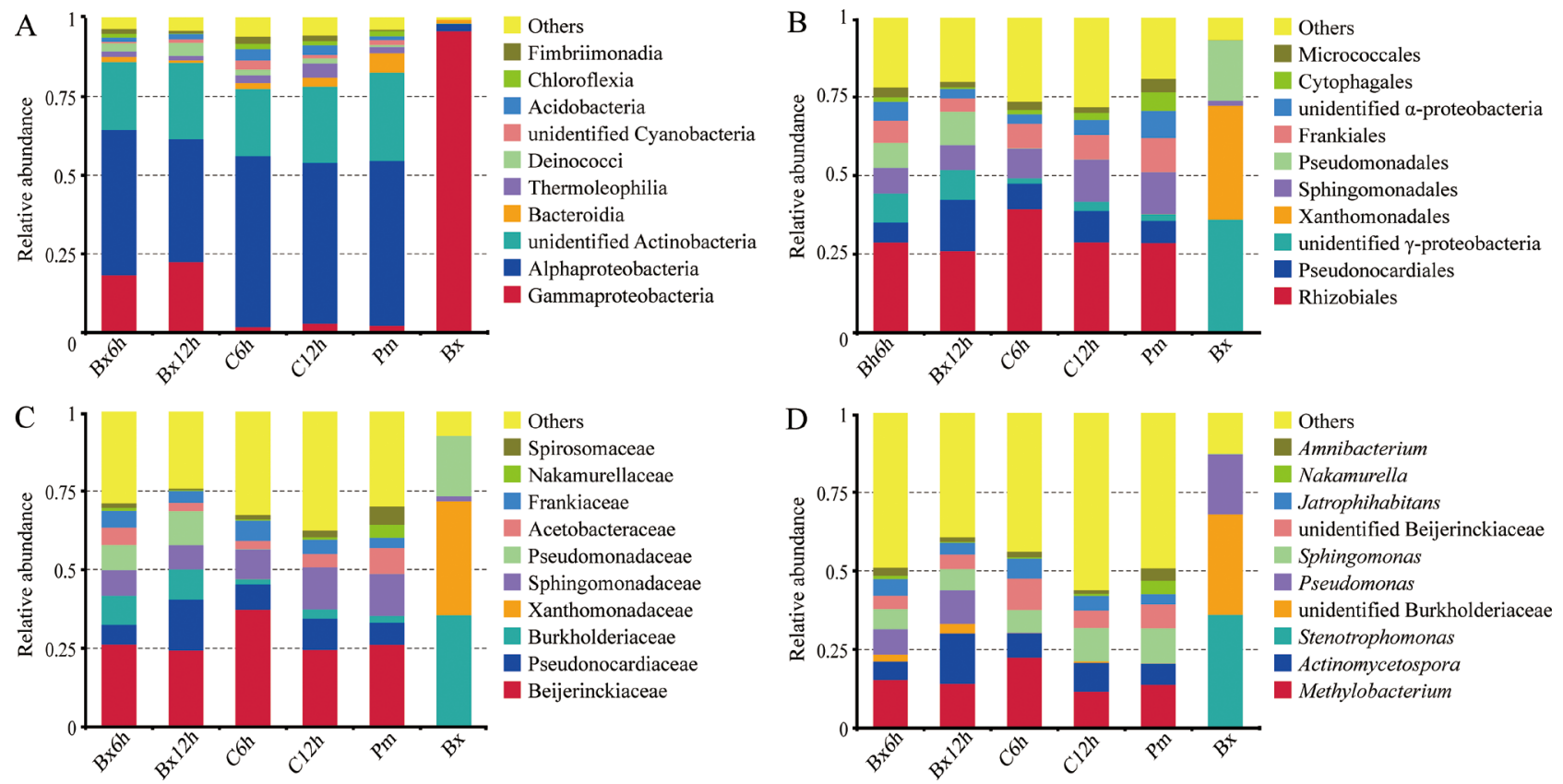

Fig. 3. Relative abundance of the top ten bacterial classes (A), orders (B), families (C) and genera (D) for each group. Bx6h, the $6 \mathrm{~h}$ pine wood nematode (PWN; Bursaphelenchus xylophilus)-inoculation group; Bx12h, the $12 \mathrm{~h}$ PWN-inoculation group; C6h, control group of Bx6h; C12h, control group of Bx12h; Pm, healthy cortex of Pinus massoniana; Bx, B. xylophilus.

For all the other groups from the wound tissues of P. massoniana (Bx6h, Bx12h, C6h, $\mathrm{C} 12 \mathrm{~h}$ and $\mathrm{Pm})$, the average number of OTUs was 684 , and the associated bacterial communities were mainly composed of Proteobacteria and Actinobacteria at the phylum level, $\alpha$-proteobacteria and unidentified Actinobacteria at the class level (Fig. 2A), and Rhizobial, Pseudonocardiales and Sphingomonadales at the order level (Fig. 2B). The most represented family was Beijerinckiaceae (Fig. 2C). However, the relative abundance at the genus level varied 
among different groups (Fig. 2D), especially between the PWN-inoculated groups and their corresponding control groups; thus, we focused on their comparative analysis at the genus level in subsequent analyses.

\section{COMPARATIVE ANALYSIS OF PWN-INOCULATION GROUPS TO CONTROL GROUPS}

In Group Bx6h, the most represented genus was Methylobacterium $(15.5 \pm 4.29 \%$ ), followed by Pseudomonas $(8.0 \pm 3.94 \%)$. With the time of inoculation prolonged (Group Bx12h), the structure and relative abundance of bacteria at the genus level showed no significant difference, except for the remarkable decrease in Nocardioides, which was detected in Group Pm but not in Group Bx.

To identify the change in bacterial communities after inoculation with PWN on the wound surface of $P$. massoniana, we compared the inoculation groups with their corresponding control groups and generated a heatmap together with relative abundance histogram at the genus level (Fig. 4). The comparative results between Groups Bx6h and C6h showed that the most abundant genus from PWN, Stenotrophomonas, completely failed to colonise the wound tissues, and the abundance of the other rep- resentative genus, Delftia, also dramatically decreased. Nevertheless, the other top represented genera of PWN (unidentified Burkholderiaceae, Pseudomonas and Serratia) were significantly enriched $(P<0.05$, MetaStat), accounting for $2.3 \pm 0.99 \%, 8.0 \pm 3.95 \%$ and $0.9 \pm$ $0.45 \%$ of the total reads in this inoculation group, respectively. Comparative analysis between Group Bx12h and the corresponding control group led to similar results except for the remarkably lower relative abundance of $\mathrm{No}$ cardioides.

LEfSe (LDA Effect Size), a software for discovering significant differences between groups, was also used, and the results indicated that both PWN-inoculation groups exhibited elevated proportions of Pseudomonas, unidentified Burkholderiaceae and Serratia (particularly Serratia liquefaciens) (Fig. 5). In addition, although with low relative abundance, other genera, such as Mesorhizobium (belonging to the family Burkholderiaceae), Rhizobacter (controversial taxonomy; our sequencing result classified it as Burkholderiaceae) and Friedmanniella, were also enriched in Group Bx6h, while Yersinia sp. Ha77 and Ewingella americana, both of which belong to Enterobacteriaceae, were enriched in Group Bx12h.

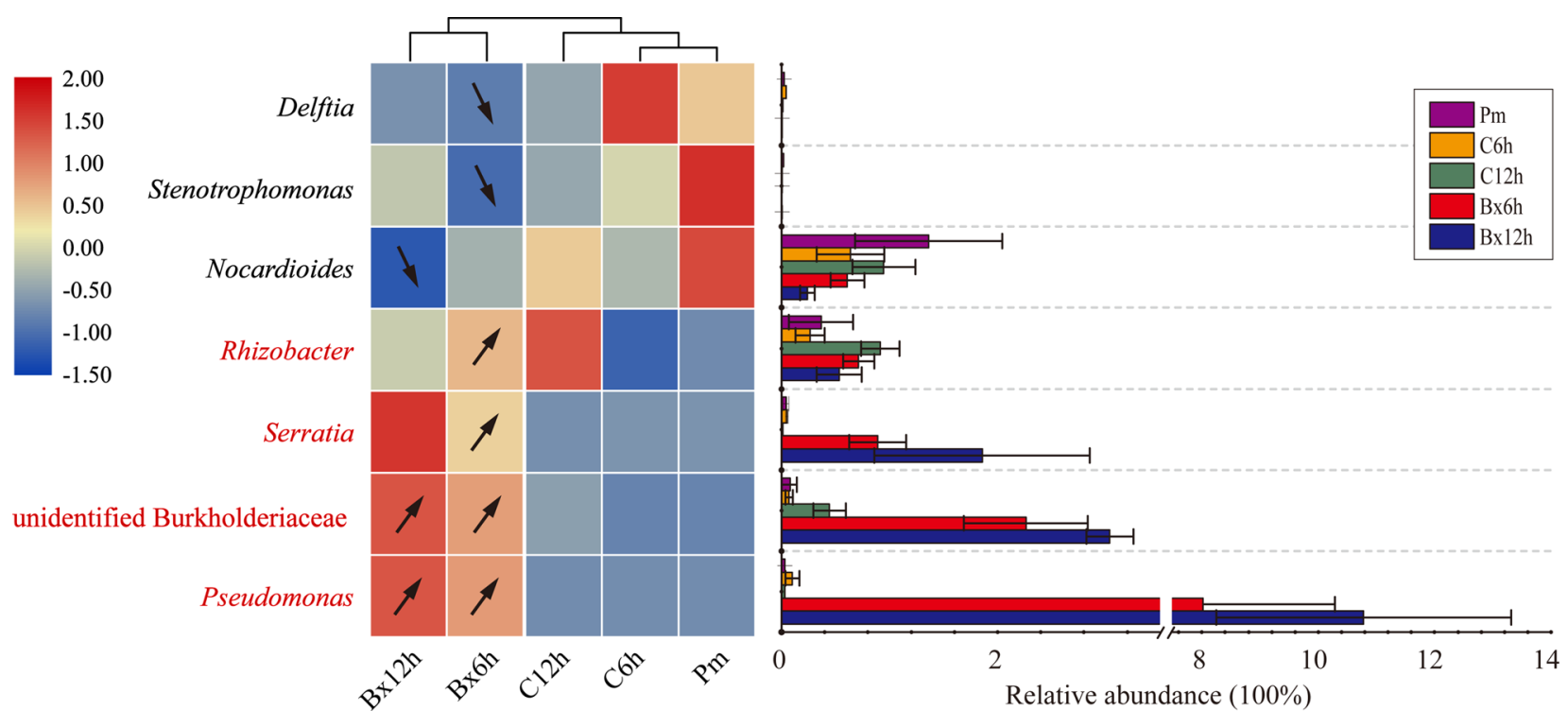

Fig. 4. Heatmap and relative abundance of genera (more than $0.5 \%$ of the total reads) with significant differences $(P<0.05$; MetaStat). The red and black font indicate the significant increase and decrease in abundance of the genera in Group Bx6h and Bx12h, respectively, compared to their control groups, and the up and down arrows in the heatmap demonstrate the specific trend in each pine wood nematode (PWN; Bursaphelenchus xylophilus)-inoculation group. The histogram shows the average relative abundance of these genera in each group $(\mathrm{n}=3)$. Error bars represent SE of the mean. Bx6h, the $6 \mathrm{~h} \mathrm{PWN}$-inoculation group; Bx12h, the $12 \mathrm{~h}$ PWN-inoculation group; $\mathrm{C} 6 \mathrm{~h}$, control group of $\mathrm{Bx} 6 \mathrm{~h} ; \mathrm{C} 12 \mathrm{~h}$, control group of $\mathrm{Bx} 12 \mathrm{~h} ; \mathrm{Pm}$, healthy cortex of Pinus massoniana; $\mathrm{Bx}$, B. xylophilus. 


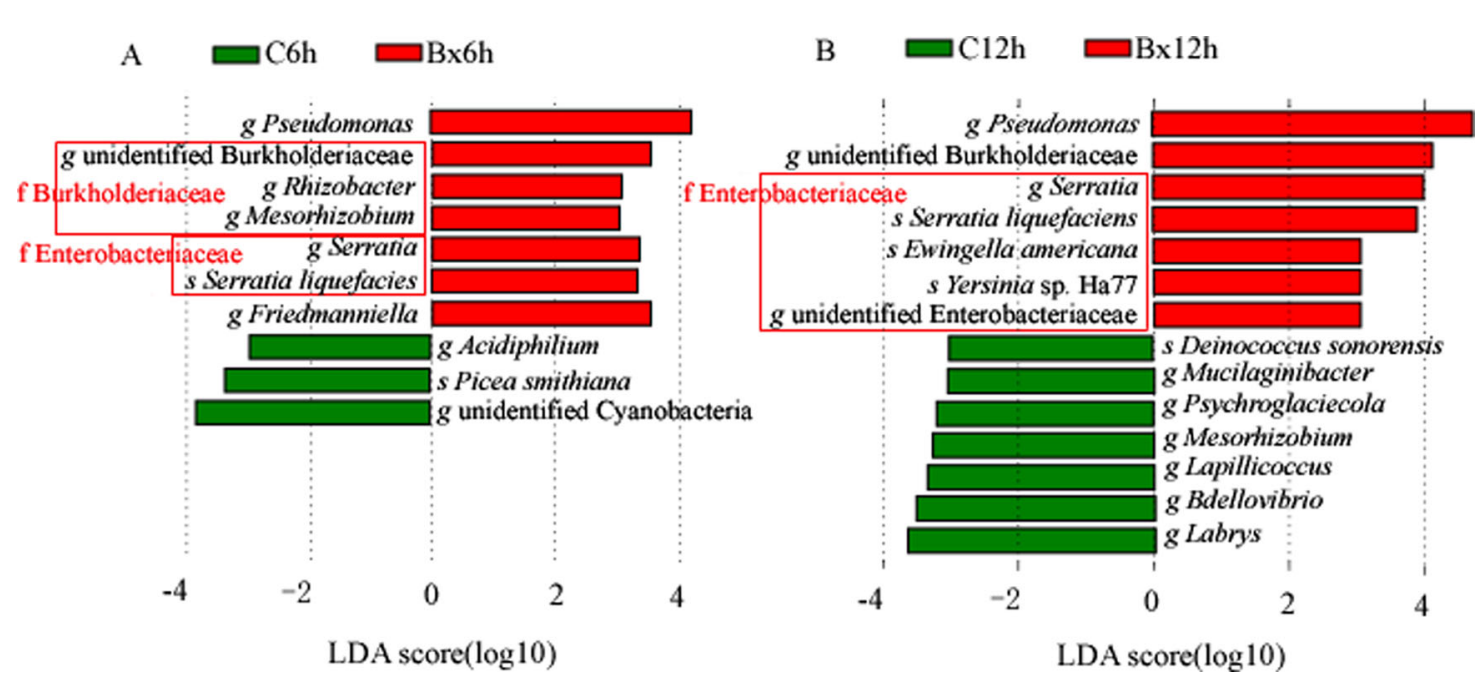

Fig. 5. Relative abundance of bacterial genera in pine wood nematode (PWN; Bursaphelenchus xylophilus)-inoculation groups (Bx6h and $\mathrm{Bx} 12 \mathrm{~h}$, red $)$ and control groups (C6h and $\mathrm{C} 12 \mathrm{~h}$, green) based on LEfSe results. A: Group Bx6h vs Group C6h; B: Group Bx12h vs Group $\mathrm{C} 12 \mathrm{~h}$. Bars represent linear discriminant analysis (LDA) scores. The threshold value was set to 3 . The lowercase letters f, $g$ and $\mathrm{s}$ in front of the bacterial names represent family, genus and species level, respectively. The genera and species of the same family were framed in red box with the family name nearby.

\section{FUNCTIONAL PREDICTION}

We used Tax4Fun based on the 16S Silva database to predict the functional diversity of the bacteria in each group. A total of 6471 functional orthologues were predicted, assigning to 43 level 2 KEGG pathways, with membrane transport, carbohydrate metabolism, amino acid metabolism and translation having greater abundance of related genes. A heatmap was made from the top 25 KEGG orthologue (KO) groups according to functional annotation and abundance, and all groups were clustered based on functional relative abundance. As expected, the functions of the bacteria associated with Group Bx were significantly different from those associated with the other groups (data not shown), with higher $\mathrm{KO}$ abundance in metabolism-related pathways such as 'enzyme families' and 'glycan biosynthesis and metabolism', environmental information processing pathways such as 'membrane transport' and 'signal transduction', and cellular processes pathways such as 'cell motility' and 'cellular community prokaryotes'. Among the other groups from the wound tissues of $P$. massoniana, the bacterial functions of Group Bx6h were more similar to those of its control group and the other two non-PWN-inoculation groups, while the bacterial functions of Group $\mathrm{Bx} 12 \mathrm{~h}$ clustered farther from all the other groups (Fig. 6). As seen from the heatmap, Group Bx12h had higher KO abundance in 'carbohydrate metabolism', 'xenobiotic biodegrada- tion and metabolism', 'amino acid metabolism', 'lipid metabolism', 'metabolism of terpenoids and polyketides', 'nucleotide metabolism', 'enzyme families', 'transport and catabolism' and 'replication and repair' pathways. In level 2 KEGG pathways, both Group Bx6h and Group $\mathrm{Bx} 12 \mathrm{~h}$ had no significant alteration in $\mathrm{KO}$ abundance compared to their control groups. However, Group Bx12h had significantly higher $\mathrm{KO}$ abundance in the 'metabolism of cofactors and vitamins' and 'nucleotide metabolism' pathways than Group Bx6h.

\section{Discussion}

Generally, the bacterial populations and diversity inside trees increase with disease progression (Xie \& Zhao, 2008), but little is known about the bacteria in wound tissues in the initial stage before the pathological response of pines to PWN invasion can be seen. Our study illustrates the changes in bacterial communities in the wound tissues of $P$. massoniana after inoculation with PWN. The results showed that the bacterial richness and diversity changed little after $6 \mathrm{~h}$ but decreased significantly after $12 \mathrm{~h}$ compared to their control groups. However, obvious alterations in some of the predominant bacteria carried by PWN occurred within $6 \mathrm{~h}$; in particular the unidentified Burkholderiaceae, Pseudomonas and Serratia ge- 


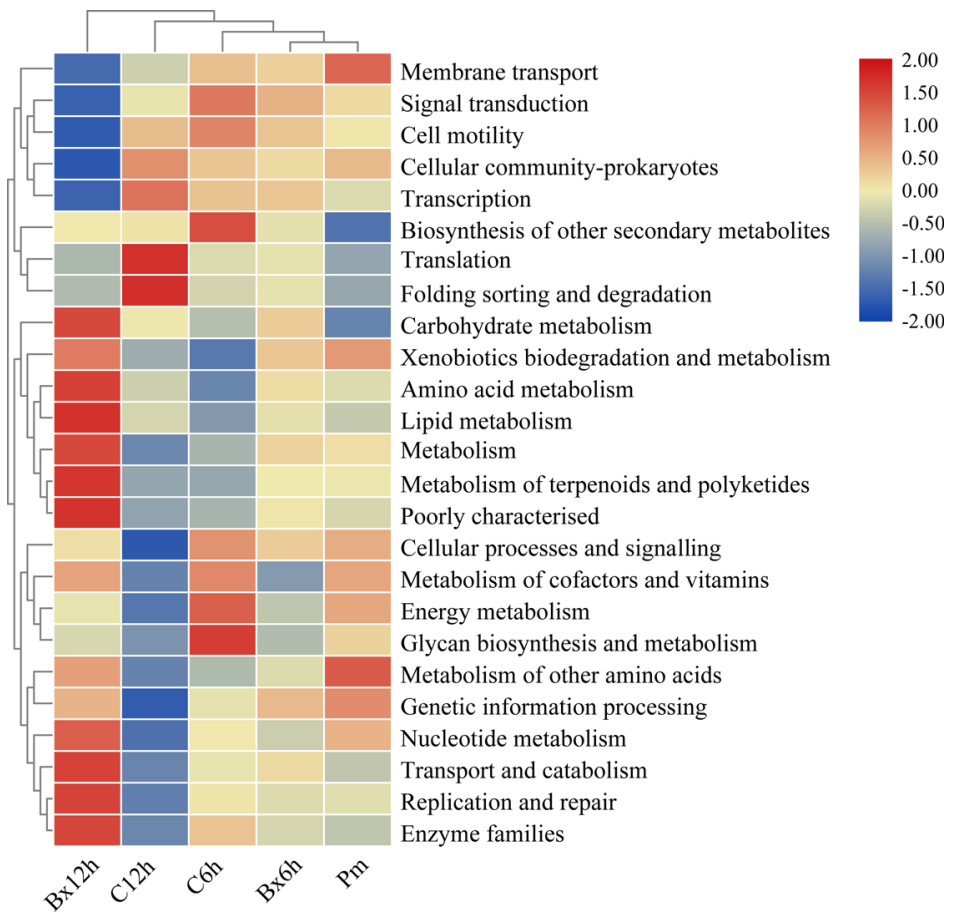

Fig. 6. Heatmap of the top $25 \mathrm{KEGG}$ orthologous (KO) groups according to the functional annotation and abundance of level 2 KEGG pathways based on Tax4Fun. Bx6h, the $6 \mathrm{~h}$ pine wood nematode (PWN; Bursaphelenchus xylophilus)-inoculation group; Bx12h, the $12 \mathrm{~h}$ PWN-inoculation group; C6h, control group of $\mathrm{Bx} 6 \mathrm{~h} ; \mathrm{C} 12 \mathrm{~h}$, control group of $\mathrm{Bx} 12 \mathrm{~h}$; Pm, healthy cortex of Pinus massoniana; $\mathrm{Bx}$, B. xylophilus.

nera were enriched but the abundance of Delftia and Stenotrophomonas were either reduced or excluded.

Unlike the increasing population dynamics of bacteria in disease stages, the bacterial colonies in the adjacent xylem of inoculation sites hardly changed after $3 \mathrm{~h}$ and were generally lower than those in the samples with disease symptoms (Roriz et al., 2011), probably because there is a barrier outside the xylem, which is the defence response of the host, leading to little change in bacterial communities in the xylem. In our study, the inoculation of PWN apparently directly changed the bacterial communities in the wound tissues of PWN, particularly after $12 \mathrm{~h}$, which may be enough time for adaptive bacteria to survive and proliferate and for host pine to eliminate unsuitable bacteria. Interestingly, Group $\mathrm{C} 12 \mathrm{~h}$ had the highest bacterial richness and diversity, indicating that even without PWN inoculation, the bacterial communities in the wound tissues might change. Although the majority of newly added bacteria remained unidentified in this group, the increasing richness was partly due to Rhizobacter, which was also observed in healthy Group Pm but with lower abundance. In addition, Rhizobacter was more abundant in both PWN-inoculation groups than Group Pm; thus, we suspect it might be beneficial for pine trees to confront injuries or resist the invasion of PWN. In support of this suggestion, Dhanasekar \& Dhandapani (2012) proposed that Rhizobacter is a nitrogen-fixing bacteria and is mainly involved in the biological control of pathogens, nutrient cycling and seedling establishment.

In Group Bx, the dominant bacterial genera were Stenotrophomonas, unidentified Burkholderiaceae, Pseudomonas, Serratia, Delftia, Novosphingobium and Chryseobacterium. Among these bacteria, Stenotrophomonas was the most dominant genus, in accordance with previous studies (Tian et al., 2010; Wu et al., 2013), and Stenotrophomonas was also detected in PWN obtained from P. massoniana. Pseudomonas, Serratia and Burkholderiaceae have been widely reported in association with PWN and are able to induce PWD symptoms (Oku et al., 1980; Han et al., 2003; Guo et al., 2006, 2007; Proença et al., 2010; Vicente et al., 2011, 2012). Meanwhile, Serratia, namely, S. marcescens, was associated with $M$. alternatus, and secondary metabolites of $S$. marcescens are able to degrade lignin (Fu, 2017). In our study, 
we also detected $S$. marcescens in PWN, but with a low proportion $(0.1 \pm 0.02 \%)$. Cheng et al. (2013) and Wang et al. (2019) found that Delftia and Novosphingobium were dominant in PWN isolated in Zhejiang Province in China, and Liu et al. (2017) identified Delftia, Pseudomonas, Stenotrophomonas and Rhizobium as the dominant species on the surface of PWN in the USA. Chryseobacterium was also reported to be one of the main species carried by the PWN from Japan (Ju et al., 2008) and the USA (Proença et al., 2014). It is plausible that bacteria carried by PWN varied in different studies because of multiple factors, such as geographic area, Pinus species, feeding fungus, isolation methodologies and surface sterilisation. To avoid eliminating the potential PWD-related bacteria, the PWN we used here were obtained without sterilisation because a considerable number of bacterial species are present on the cuticle surface of PWN (Roriz et al., 2011; Vicente et al., 2011), and superficially associated bacteria can also potentially be involved in PWD (Li, 2008).

In Group Pm, the bacterial community was dominated by Proteobacteria and Actinobacteria at the phylum level. The top three genera were Methylobacterium, Sphingomonas and unidentified Beijerinckiaceae, all of which belong to Proteobacteria. A recent study also reported that the most dominant endophytic bacteria in healthy, as well as in diseased, P. massoniana is Proteobacteria (Li et al., 2018). However, the corresponding genera were different, and the second most dominant phylum was Bacteroides, which ranked third in our study. These differences could be explained by differences in sampling location, sampling area and tree age. In addition, several studies have confirmed the existence of endophytic bacteria in pine trees, with Methylobacterium being the major genus (Pirttilä et al., 2000, 2008), possibly simply because it is a beneficial endophytic bacteria for the growth of pine seedlings (Pohjanen et al., 2014).

After inoculation with PWN, unidentified Burkholderiaceae, Pseudomonas and Serratia (especially S. liquefaciens) were introduced to the wound surface together with PWN and significantly enriched within at least $6 \mathrm{~h}$, while interestingly the most dominant genus, Stenotrophomonas, could not colonise wound tissues and the abundance of one of the representative genera, Delftia, was reduced, indicating that unidentified Burkholderiaceae, Pseudomonas and Serratia adapted to the environment containing secondary metabolites from the host but Stenotrophomonas and Delftia did not. For the above result, we may doubt that the increases in Burkholderiaceae, Pseudomonas and Serratia resulted from the in- troduction of PWN, but the abundance of these bacteria also increased in Group Bx12h compared to Group Bx6h, confirming that they were indeed enriched in the wound tissues and may play a role in the initial infection activities. In summary, these bacteria could be considered as biomarkers to understand the development of PWD in this specific stage.

As terpenoids (especially $\alpha$-pinene) are defence compounds of pine trees and are detrimental to the reproduction of the PWN (Kong et al., 2007), $\alpha$-pinene degradation is important for the survival and invasion of PWN. It was reported that Pseudomonas is one of the main strains capable of degrading $\alpha$-pinene; it not only survives the stress of $\alpha$-pinene but also utilises $\alpha$-pinene and other secondary metabolites of pine, including benzoate, as a carbon source for growth, whilst Stenotrophomonas cannot survive under the stress of benzoate and grows slowly in LB medium containing $\alpha$-pinene (Cheng et al., 2013). A recent study observed similar results; the abundance of Pseudomonas sp. carried by PWN increased, while the abundance of Stenotrophomonas and Delftia spp. decreased when PWN was fumigated with $\alpha$-pinene (Wang et al., 2019), which may explain the enrichment of Pseudomonas and the reduction or even exclusion of Delftia and Stenotrophomonas in our study. However, we could not ignore the fact that one species of Stenotrophomonas, namely, S. maltophilia, was related to the strong virulence of PWN (Wu et al., 2013), and when S. maltophilia NSPmBx03 was treated with PWN, many cell wall degradation-related and detoxificationrelated genes, including pectate lyase, glutathione $\mathrm{S}$ transferase, ATP-binding cassette transporter and cytochrome P450, might be upregulated, and the virulence of PWN might be enhanced (He et al., 2016). In addition, some Stenotrophomonas spp. were reported as being able to degrade aromatic compounds and other xenobiotics (Mangwani et al., 2014; Tiwari et al., 2016), which seems beneficial for PWN to invade its host. However, other strains of $S$. maltophilia might inhibit PWN hatching (Tian et al., 2015) or possess nematicidal activity against PWN (Huang et al., 2009), leading to the opposite result. A similar controversy was also presented by Serratia: many species exhibited high toxicity against $\mathrm{PWN}$ in vitro (Paiva et al., 2013) but some were able to produce copious cellulose, form biofilms, resist reactive oxygen species, tolerate growth in the presence of xenobiotic/organic compounds and not only colonise themselves (Nascimento et al., 2018; Vicente et al., 2012, 2013a, 2016b) but also assist PWN survival under prolonged oxidative stress con- 
ditions (Vicente et al., 2013b, 2016b). Considering these contradictory results on different strains within the same genera, the role of these bacteria in PWN and PWD needs more investigation. Interestingly, within the Serratia genus, S. liquefaciens was reported for the first time in our study to be related to PWN. It was relatively abundant (3.3\%) in Group Bx and enriched in the wound tissues after $6 \mathrm{~h}$ of PWN inoculation. Although its detailed role in PWN remains to be further studied, this species has been described to produce volatile organic compounds and thus could promote plant growth and attract Caenorhabditis elegans (Grewal \& Wright, 1992; Wang, 2018).

To predict the functions of bacteria, we used the Tax4Fun approach. Because of the limitation of this method, we could not identify the exact role of each bacterium, but the results of this section could suggest that the alterations in bacterial composition potentially lead to shifts in their functional pathways. This result was more obvious in the longer inoculation time group (Group $\mathrm{Bx} 12 \mathrm{~h}$ ) in our study and was reflected mainly in the increase in $\mathrm{KO}$ abundance in multiple metabolism-related pathways such as 'carbohydrate metabolism', 'xenobiotics biodegradation and metabolism', 'amino acid metabolism', 'lipid metabolism', and 'metabolism of terpenoids and polyketides', which potentially indicates that related bacteria may play a role in the invasion of PWN at $12 \mathrm{~h}$. Further studies are needed to determine the specific role of these bacteria in pine trees in vivo.

\section{Acknowledgements}

The authors acknowledge the financial support of the National Key Research and Development Program of China (2017YFD0600105) and the Natural Science Foundation of Fujian Province (2019J01368). We also thank our colleagues Lizhen Feng, Zhang Song, Xiaozhen Ye, Qinhua Zhang, Quanzhu Chen and Hongyi Liu for useful discussions.

\section{References}

Aikawai, T. \& Tiogashi, K. (1998). An effect of pine volatiles on departure of Bursaphelenchus xylophilus (Nematoda: Aphelenchoididae) from Monochamus alternatus (Coleoptera: Cerambycidae). Applied Entomology and Zoology 33, 231-237. DOI: $10.1303 /$ aez.33.231

Alves, M., Pereira, A., Matos, P., Henriques, J., Vicente, C., Aikawa, T., Hasegawa, K., Nascimento, F., Mota, M., Correia, A. et al. (2016). Bacterial community associated to the pine wilt disease insect vectors Monochamus galloprovincialis and Monochamus alternatus. Scientific Reports 6, 23908. DOI: 10.1038/srep23908

Aßhauer, K.P., Wemheuer, B., Daniel, R. \& Meinicke, P. (2015). Tax4Fun: predicting functional profiles from metagenomic 16S rRNA data. Bioinformatics 31, 2882-2884. DOI: 10. 1093/bioinformatics/btv287

Caporaso, J.G., Kuczynski, J., Stombaugh, J., Bittinger, K., Bushman, F.D., Costello, E.K., Fierer, N., Peña, A.G., Goodrich, J.K., Gordon, J.I. et al. (2010). QIIME allows analysis of high-throughput community sequencing data. Nature Methods 7, 335-336. DOI: 10.1038/nmeth.f.303

Cheng, X.Y., Tian, X.L., Wang, Y.S., Lin, R.M., Mao, Z.C., Chen, N. \& Xie, B.Y. (2013). Metagenomic analysis of the pinewood nematode microbiome reveals a symbiotic relationship critical for xenobiotics degradation. Scientific Reports 3, 1869. DOI: 10.1038/srep01869

Dhanasekar, R. \& Dhandapani, R. (2012). Effect of biofertilizers on the growth of Helianthus annuus. International Journal of Plant, Animal and Environmental Sciences 2, 143-147.

Edgar, R.C. (2013). UPARSE: highly accurate OTU sequences from microbial amplicon reads. Nature Methods 10, 996-998. DOI: $10.1038 /$ nmeth.2604

Edgar, R.C., Haas, B.J., Clemente, J.C., Quince, C. \& Knight, R. (2011). UCHIME improves sensitivity and speed of chimera detection. Bioinformatics 27, 2194-2200. DOI: 10. 1093/bioinformatics/btr381

Edwards, O.R. \& Linit, M.J. (1992). Transmission of Bursaphelenchus xylophilus through oviposition wounds of Monochamus carolinensis (Coleoptera: Cerambycidae). Journal of $\mathrm{Ne}$ matology 24, 133-139.

Fu, H.J. (2017). [Studies on bacteria diversity in the gut of Monochamus alternatus Hope and lignin-degradation characteristics of Serratia marcescens.] M.Sc. Thesis, Fujian Agriculture and Forestry University, Fuzhou, China.

Fukuda, K., Hogetsu, T. \& Suzuki, K. (1992). Cavitation and cytological changes in xylem of pine seedlings inoculated with virulent and avirulent isolates of Bursaphelenchus xylophilus and B. mucronatus. Journal of the Japanese Forestry Society 74, 289-299. DOI: 10.11519/jjfs1953.74.4_289

Grewal, P.S. \& Wright, D.J. (1992). Migration of Caenorhabditis elegans (Nematoda: Rhabditidae) larvae towards bacteria and the nature of the bacterial stimulus. Fundamental and Applied Nematology 15, 159-166.

Guo, D.S., Zhao, B.G. \& Li, R.G. (2006). [Effect of pinewood nematode on the propagation and pathogenicity of its carrying bacterial strain.] Chinese Journal of Applied and Environmental Biology 12, 523-527. DOI: 10.3321/j.issn:1006-687X. 2006.04.018

Guo, Q.Q., Guo, D.S., Zhao, B.G., Xu, J. \& Li, R.G. (2007). Two cyclic dipeptides from Pseudomonas fluorescens GcM5-1A carried by the pine wood nematode and their toxicities to Japanese black pine suspension cells and seedlings in vitro. Journal of Nematology 39, 243-247. 
Han, Z.M., Hong, Y.D. \& Zhao, B.G. (2003). A study on pathogenicity of bacteria carried by pine wood nematodes. Journal of Phytopathology 151, 683-689. DOI: 10.1046/j. 1439-0434.2003.00790.x

He, L.X., Wu, X.Q., Xue, Q. \& Qiu, X.W. (2016). Effects of endobacterium (Stenotrophomonas maltophilia) on pathogenesis-related gene expression of pine wood nematode (Bursaphelenchus xylophilus) and pine wilt disease. International Journal of Molecular Sciences 17, 778. DOI: 10.3390/ ijms 17060778

Huang, X., Liu, J., Ding, J., He, Q., Xiong, R. \& Zhang, K. (2009). The investigation of nematocidal activity in Stenotrophomonas maltophilia $\mathrm{G} 2$ and characterization of a novel virulence serine protease. Canadian Journal of Microbiology 55, 934-942. DOI: 10.1139/w09-045

Jin, G. (2007). [Research of programmed cell death in interaction between Pinus thunbergii and Bursaphelenchus xylophilus.] Ph.D. Thesis, Nanjing Forestry University, Nanjing, China.

Ju, Y.W., Xie, L.Q., Yang, X.Y. \& Zhao, B.G. (2008). [Varieties of bacteria carried by pine wood nematode from different sources.] Journal of Northeast Forestry University 36, 84-85. DOI: 10.3969/j.issn.1000-5382.2008.05.032

Kawazu, K., Zhang, H. \& Kanzaki, H. (1996). Accumulation of benzoic acid in suspension cultured cells of Pinus thunbergii Parl. in response to phenylacetic acid administration. Bioscience, Biotechnology and Biochemistry 60, 1410-1412. DOI: $10.1271 /$ bbb.60.1410

Kiyohara, T. \& Tokushige, Y. (1971). Inoculation experiments of a nematode, Bursaphelenchus sp., onto pine trees. Journal of the Japanese Forestry Society 53, 210-218. DOI: 10.11519/ jjfs1953.53.7_210

Kong, J.O., Park, I.K., Choi, K.S., Shin, S.C. \& Ahn, Y.J. (2007). Nematicidal and propagation activities of thyme red and white oil compounds toward Bursaphelenchus xylophilus (Nematoda: Parasitaphelenchidae). Journal of Nematology 39, 237.

Lee, S.M., Choo, H.Y., Park, N.C., Moon, Y.S. \& Kim, J.B. (1990). Nematodes and insects associated with dead trees, and pine wood nematode detection in Monochamus alternatus. Korean Journal of Applied Entomology 29, 14-19.

$\mathrm{Li}, \mathrm{H}$. (2008). Identification and pathogenicity of Bursaphelenchus species (Nematoda: Parasitaphelenchidae). Ph.D. Thesis, Ghent University, Ghent, Belgium.

Li, Y. \& Ye, J.R. (2008). [Death manner of host parenchymatous cells in infecting early stage of pine wilt disease.] Scientia Silvae Sinicae 44, 95-98. DOI: 10.11707/j.1001-7488.20080418

Li, Y., Yao, Q.Z., Xing, Z., Wu, C.F. \& Zhang, L.Q. (2018). Responses of endophytic microbial community in masson pine to the early stage of pine wilt disease. Journal of Botanical Sciences 7, 61-69.

Linit, M.J. (1990). Transmission of pinewood nematode through feeding wounds of Monochamus carolinensis (Coleoptera: Cerambycidae). Journal of Nematology 22, 231-236.
Linit, M.J., Kondo, E. \& Smith, M.T. (1983). Insects associated with the pinewood nematode, Bursaphelenchus xylophilus (Nematoda: Aphelenchoididae), in Missouri. Environmental Entomology 12, 467-470. DOI: 10.1093/ee/12.2.467

Liu, K.C., Zeng, F.L., Ben, A.L. \& Han, Z.M. (2017). Pathogenicity and repulsion for toxin-producing bacteria of dominant bacteria on the surface of American pine wood nematodes. Journal of Phytopathology 165, 580-588. DOI: 10. 1111/jph.12595

Lutz, K.A., Wang, W., Zdepski, A. \& Michael, T.P. (2011). Isolation and analysis of high quality nuclear DNA with reduced organellar DNA for plant genome sequencing and resequencing. BMC Biotechnology 11, 54. DOI: 10.1186/ 1472-6750-11-54

Mamiya, Y. (1975). Behavior of pine wood nematodes in pine wood in early stages of the disease development. Transactions of the Meeting of the Japanese Forestry Society 86, 285-286.

Mamiya, Y. (1983). Pathology of the pine wilt disease caused by Bursaphelenchus xylophilus. Annual Review of Phytopathology 21, 201-220. DOI: 10.1146/annurev.py.21. 090183.001221

Mamiya, Y. \& Enda, N. (1972). Transmission of Bursaphelenchus lignicolus (Nematoda: Aphelenchoididae) by Monochamus alternatus (Coleoptera: Cerambycidae). Nematologica 18, 159-162. DOI: 10.1163/187529272x00395

Mangwani, N., Shukla, S.K., Kumari, S., Rao, T.S. \& Das, S. (2014). Characterization of Stenotrophomonas acidaminiphila NCW-702 biofilm for implication in the degradation of polycyclic aromatic hydrocarbons. Journal of Applied Microbiology 117, 1012-1024. DOI: 10.1111/jam.12602

Martin, M. (2011). Cutadapt removes adapter sequences from high-throughput sequencing reads. EMBnet.journal 17, 10-12. DOI: 10.14806/ej.17.1.200

Nascimento, F., Vicente, C., Cock, P., Tavares, M., Rossi, M., Hasegawa, K. \& Mota, M. (2018). From plants to nematodes: Serratia grimesii BXF1 genome reveals an adaptation to the modulation of multi-species interactions. Microbial Genomics 4, 1-13. DOI: 10.1099/mgen.0.000178

Naves, P., Mota, M., Pires, J., Penas, A.C., Sousa, E., Bonifácio, L. \& Bravo, M.A. (2001). Bursaphelenchus xylophilus (Nematoda; Aphelenchoididae) associated with Monochamus galloprovincialis (Coleoptera; Cerambycidae) in Portugal. Nematology 3, 89-91. DOI: 10.1163/156854101300106937

Nickle, W.R., Golden, A.M., Mamiya, Y. \& Wergin, W.P. (1981). On the taxonomy and morphology of the pine wood nematode, Bursaphelenchus xylophilus (Steiner \& Buhrer 1934) Nickle 1970. Journal of Nematology 13, 385-392.

Ning, T., Fang, Y.L., Tang, J. \& Sun, J.H. (2004). [Advances in research on Bursaphelenchus xylophilus and its key vector Monochamus spp.] Entomological Knowledge 41, 97-104. DOI: 10.3969/j.issn.0452-8255.2004.02.001

Oku, H., Shiraishi, T. \& Kurozumi, S. (1979). Participation of toxin in wilting of Japanese pines caused by a nematode. Naturwissenschaften 66, 210. DOI: 10.1007/bf00366031 
Oku, H., Shiraishi, T., Ouchi, S., Kurozumi, S. \& Ohta, H. (1980). Pine wilt toxin, the metabolite of a bacterium associated with a nematode. Naturwissenschaften 67, 198-199. DOI: $10.1007 / \mathrm{bf01086307}$

Paiva, G., Proenca, D.N., Francisco, R., Verissimo, P., Santos, S.S., Fonseca, L., Abrantes, I.M.O. \& Morais, P.V. (2013). Nematicidal bacteria associated to pinewood nematode produce extracellular proteases. PLOS ONE 8, e79705. DOI: 10. 1371/journal.pone.0079705

Pirttilä, A.M., Laukkanen, H., Pospiech, H., Myllyla, R. \& Hohtola, A. (2000). Detection of intracellular bacteria in the buds of Scotch pine (Pinus sylvestris L.) by in situ hybridization. Applied and Environmental Microbiology 66, 3073-3077. DOI: 10.1128/aem.66.7.3073-3077.2000

Pirttilä, A.M., Podolich, O., Koskimäki, J.J., Hohtola, E. \& Hohtola, A. (2008). Role of origin and endophyte infection in browning of bud-derived tissue cultures of Scots pine (Pinus sylvestris L.). Plant Cell, Tissue and Organ Culture 95, 47-55. DOI: $10.1007 / \mathrm{s} 11240-008-9413-\mathrm{x}$

Pohjanen, J., Koskimaki, J.J., Sutela, S., Ardanov, P., Suorsa, M., Niemi, K., Sarjala, T., Haggman, H. \& Pirttila, A.M. (2014). Interaction with ectomycorrhizal fungi and endophytic Methylobacterium affects nutrient uptake and growth of pine seedlings in vitro. Tree Physiology 34, 993-1005. DOI: 10.1093/treephys/tpu062

Proença, D.N., Francisco, R., Santos, C.V., Lopes, A., Fonseca, L., Abrantes, I.M.O. \& Morais, P.V. (2010). Diversity of bacteria associated with Bursaphelenchus xylophilus and other nematodes isolated from Pinus pinaster trees with pine wilt disease. PLOS ONE 5, e15191. DOI: 10.1371/journal. pone.0015191

Proença, D.N., Fonseca, L., Powers, T.O., Abrantes, I.M.O. \& Morais, P.V. (2014). Diversity of bacteria carried by pinewood nematode in USA and phylogenetic comparison with isolates from other countries. PLOS ONE 9, e105190. DOI: 10.1371/ journal.pone.0105190

Proença, D.N., Francisco, R., Kublik, S., Schöler, A., Vestergaard, G., Schloter, M. \& Morais, P.V. (2017a). The microbiome of endophytic, wood colonizing bacteria from pine trees as affected by pine wilt disease. Scientific Reports 7, 4205. DOI: 10.1038/s41598-017-04141-6

Proença, D.N., Grass, G. \& Morais, P.V. (2017b). Understanding pine wilt disease: roles of the pine endophytic bacteria and of the bacteria carried by the disease-causing pinewood nematode. MicrobiologyOpen 6, e00415. DOI: 10.1002/mbo3.415

Quast, C., Pruesse, E., Yilmaz, P., Gerken, J., Schweer, T., Yarza, P., Peplies, J. \& Glöckner, F.O. (2012). The SILVA ribosomal RNA gene database project: improved data processing and web-based tools. Nucleic Acids Research 41, D590-D596. DOI: $10.1093 /$ nar/gks1219

Roriz, M., Santos, C. \& Vasconcelos, M.W. (2011). Population dynamics of bacteria associated with different strains of the pine wood nematode Bursaphelenchus xylophilus after inoculation in maritime pine (Pinus pinaster). Experimental
Parasitology 128, 357-364. DOI: 10.1016/j.exppara.2011.04. 008

Segata, N., Izard, J., Waldron, L., Gevers, D., Miropolsky, L., Garrett, W.S. \& Huttenhower, C. (2011). Metagenomic biomarker discovery and explanation. Genome Biology 12, R60. DOI: 10.1186/gb-2011-12-6-r60

Sriwati, R., Takemoto, S. \& Futai, K. (2008). The relationship between the pinewood nematode (PWN) and fungi cohabiting in pine trees inoculated with the PWN. In: Mota, M.M. \& Vieira, P. (Eds). Pine wilt disease: a worldwide threat to forest ecosystems. Dordrecht, The Netherlands, Springer, pp. 101114. DOI: 10.1007/978-1-4020-8455-3_9

Su, S.R., Li, Y.Q. \& Ye, J.R. (2008). [Histopathological changes in the resistant pine trees inoculated by pine wood nematode.] Journal of Nanjing Forestry University (Natural Sciences Edition) 32, 95-98. DOI: 10.3969/j.issn.1000-2006.2008.03. 023

Tamura, H. (1984). Early development of Bursaphlenchus xylophilus (Nematoda: Aphelenchoididae) population in the inoculated branches of pine seedlings. Applied Entomology and Zoology 19, 125-129. DOI: 10.1303/aez.19.125

Tan, J.J. \& Feng, Z.X. (2004). Population dynamics of pine wood nematode and its accompanying bacterium in the host. Scientia Silvae Sinicae 40, 110-114. DOI: 10.11707/j.10017488.20040619

Tian, X.-J., Xiang, Y., Ye, J.R., Wu, X.-Q. \& Fang, X. (2015). The effect of endobacteria on the development and virulence of the pine wood nematode, Bursaphelenchus xylophilus. Nematology 17, 581-589. DOI: 10.1163/15685411-00002892

Tian, X.L., Zhang, Q.L., Chen, G.H., Mao, Z.C., Yang, J.R. \& Xie, B.Y. (2010). [Metagenomic analysis of bacterial diversity associated with pine wood nematodes.] Acta Microbiologica Sinica 50, 909-916. DOI: 10.13343/j.cnki.wsxb.2010. 07.014

Tiwari, B., Manickam, N., Kumari, S. \& Tiwari, A. (2016). Biodegradation and dissolution of polyaromatic hydrocarbons by Stenotrophomonas sp. Bioresource Technology 216, 1102-1105. DOI: 10.1016/j.biortech.2016.06.047

Vicente, C.S.L., Nascimento, F.X., Barbosa, P., Ke, H.-M., Tsai, I.J., Hirao, T., Cock, P.J.A., Kikuchi, T., Hasegawa, K. \& Mota, M. (2016a). Evidence for an opportunistic and endophytic lifestyle of the Bursaphelenchus xylophilusassociated bacteria Serratia marcescens PWN146 isolated from wilting Pinus pinaster. Microbial Ecology 72, 669-681. DOI: 10.1007/s00248-016-0820-y

Vicente, C.S.L., Nascimento, F., Espada, M., Mota, M. \& Oliveira, S. (2011). Bacteria associated with the pinewood nematode Bursaphelenchus xylophilus collected in Portugal. Antonie van Leeuwenhoek 100, 477-481. DOI: 10.1007/ s10482-011-9602-1

Vicente, C.S.L., Nascimento, F., Espada, M., Barbosa, P., Mota, M., Glick, B.R. \& Oliveira, S. (2012). Characterization of bacteria associated with pinewood nematode Bursaphelen- 
chus xylophilus. PloS ONE 7, e46661. DOI: 10.1371/journal. pone.0046661

Vicente, C.S.L., Nascimento, F.X., Espada, M., Barbosa, P., Hasegawa, K., Mota, M. \& Oliveira, S. (2013a). Characterization of bacterial communities associated with the pine sawyer beetle Monochamus galloprovincialis, the insect vector of the pinewood nematode Bursaphelenchus xylophilus. FEMS Microbiology Letters 347, 130-139. DOI: 10.1111/1574-6968. 12232

Vicente, C.S.L., Ikuyo, Y., Mota, M. \& Hasegawa, K. (2013b). Pinewood nematode-associated bacteria contribute to oxidative stress resistance of Bursaphelenchus xylophilus. BMC Microbiology 13, 1-8. DOI: 10.1186/1471-2180-13-299

Vicente, C.S.L., Nascimento, F.X., Ikuyo, Y., Cock, P.J.A., Mota, M. \& Hasegawa, K. (2016b). The genome and genetics of a high oxidative stress tolerant Serratia sp. LCN16 isolated from the plant parasitic nematode Bursaphelenchus xylophilus. BMC Genomics, 17. DOI: 10.1186/s12864-016-2626-1

Wang, M.J. (2018). [Isolation, screening and potential evaluation of biocontrol.] M.Sc. Thesis, Wuhan, China, Huazhong Agricultural University.

Wang, X., Yu, Y.X., Ge, J.J., Xie, B.Y., Zhu, S.F. \& Cheng, X.Y. (2019). Effects of $\alpha$-pinene on the pinewood nematode (Bursaphelenchus xylophilus) and its symbiotic bacteria. PLoS ONE 14, e0221099. DOI: 10.1371/journal.pone.0221099

White, J.R., Nagarajan, N. \& Pop, M. (2009). Statistical methods for detecting differentially abundant features in clinical metagenomic samples. PLoS Computational Biology 5, e1000352. DOI: 10.1371/journal.pcbi.1000352

Wu, X.Q., Yuan, W.M., Tian, X.J., Fan, B., Fang, X., Ye, J.R. \& Ding, X.L. (2013). Specific and functional diversity of endophytic bacteria from pine wood nematode Bursaphelenchus Xylophilus with different virulence. International Journal of Biological Sciences 9, 34-44. DOI: 10.7150/ijbs.5071

Xie, L.Q. \& Zhao, B.G. (2008). Post-inoculation population dynamics of Bursaphelenchus xylophilus and associated bacteria in pine wilt disease on Pinus thunbergii. Journal of Phy- topathology 156, 385-389. DOI: 10.1111/j.1439-0434.2007. 01357.x

Xie, W.F., Huang, A.Z., Li, H.M., Feng, L.Z., Zhang, F.P. \& Guo, W.S. (2017a). Identification and comparative analysis of microRNAs in Pinus massoniana infected by Bursaphelenchus xylophilus. Plant Growth Regulation 83, 223-232. DOI: 10.1007/s10725-016-0221-8

Xie, W.F., Li, H.M., Huang, A.Z., Feng, L.Z. \& Zhang, F.P. (2017b). [The dynamic change of gene expression from Pinus massoniana in response to Bursaphelenchus xylophilus isolation.] Fujian Journal of Agricultural Sciences 32, 403409. DOI: 10.19303/j.issn.1008-0384.2017.04.010

Yin, Y.H., Guo, D.S., Zhao, B.G., Du, X.H., Zhao, Z.T. \& Li, R.G. (2007). [Primary study on the toxins secreted by Pseudomonas fluorescens GcM5-1A carried by pine wood nematodes.] Chinese Bulletin of Botany 24, 147-153. DOI: 10.3969/j.issn.1674-3466.2007.02.004

Zhang, Y., Wu, X.Q., Wang, Y. \& Ye, J.R. (2007). [Resistance determination and histopathological observation of induced Pinus densiflora families from Japan to Bursaphelenchus xylophilus.] Journal of Nanjing Forestry University (Natural Sciences Edition) 31, 110-114. DOI: 10.3969/j.issn.10002006.2007.04.025

Zhao, B.G. (2008). Bacteria carried by the pine wood nematode and their symbiotic relationship with the nematode. In: Zhao, B.G., Futai, K., Sutherland, J.R. \& Takeuchi, Y. (Eds). Pine wilt disease. Tokyo, Japan, Springer, pp. 264-273. DOI: 10. 1007/978-4-431-75655-2_27

Zhao, B.G., Guo, D.S., Gao, R. \& Guo, J. (2000). [A preliminary study on the relationship between the bacterium isolate B619 and pine wilt disease.] Journal of Nanjing Forestry University (Natural Sciences Edition) 24, 72-74. DOI: 10.3969/j.issn. 1000-2006.2000.04.017

Zhao, B.G., Wang, H.L., Han, S.F. \& Han, Z.M. (2003). Distribution and pathogenicity of bacteria species carried by Bursaphelenchus xylophilus in China. Nematology 5, 899906. DOI: $10.1163 / 156854103773040817$ 